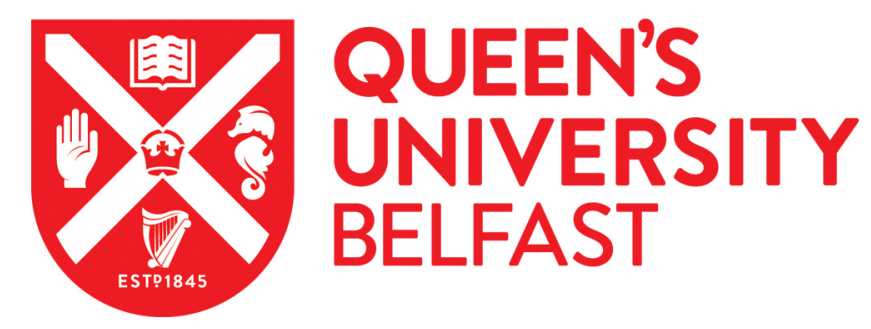

\title{
Isolated vibrational wavepackets in D-2(+): Defining superposition conditions and wavepacket distinguishability
}

Bryan, W. A., McKenna, J., English, E. M. L., Wood, J., Calvert, C. R., Torres, R., Murphy, D., Turcu, I. C. E., Collier, J. L., McCann, J., Williams, I., \& Newell, W. R. (2007). Isolated vibrational wavepackets in D-2(+): Defining superposition conditions and wavepacket distinguishability. Physical Review A, 76(5), [053402]. https://doi.org/10.1103/PhysRevA.76.053402

Published in:

Physical Review A

Queen's University Belfast - Research Portal:

Link to publication record in Queen's University Belfast Research Portal

\section{General rights}

Copyright for the publications made accessible via the Queen's University Belfast Research Portal is retained by the author(s) and / or other copyright owners and it is a condition of accessing these publications that users recognise and abide by the legal requirements associated with these rights.

Take down policy

The Research Portal is Queen's institutional repository that provides access to Queen's research output. Every effort has been made to ensure that content in the Research Portal does not infringe any person's rights, or applicable UK laws. If you discover content in the Research Portal that you believe breaches copyright or violates any law, please contact openaccess@qub.ac.uk. 


\title{
Isolated vibrational wavepackets in $\mathrm{D}_{2}^{+}$: Defining superposition conditions and wavepacket distinguishability
}

\author{
W. A. Bryan, ${ }^{1, *}$ J. McKenna, ${ }^{2}$ E. M. L. English, ${ }^{1}$ J. Wood, ${ }^{1}$ C. R. Calvert, ${ }^{2}$ R. Torres, ${ }^{3}$ D. S. Murphy, ${ }^{2}$ I. C. E. Turcu, ${ }^{4}$ \\ J. L. Collier, ${ }^{4}$ J. F. McCann, ${ }^{2}$ I. D. Williams, ${ }^{2, \dagger}$ and W. R. Newell, ${ }^{1, \$}$ \\ ${ }^{1}$ Department of Physics and Astronomy, University College London, Gower Street, London WC1E 6BT, United Kingdom \\ ${ }^{2}$ Department of Pure and Applied Physics, Queen's University Belfast, Belfast BT7 1NN, United Kingdom \\ ${ }^{3}$ Blackett Laboratory, Imperial College London, Prince Consort Road, London SW7 2BW, United Kingdom \\ ${ }^{4}$ Central Laser Facility, Rutherford Appleton Laboratory, Chilton, Didcot, Oxon OX11 OQX, United Kingdom
}

(Received 26 April 2007; published 5 November 2007)

\begin{abstract}
Tunnel ionization of room-temperature $\mathrm{D}_{2}$ in an ultrashort (12 femtosecond) near infrared (800 nm) pump laser pulse excites a vibrational wavepacket in the $\mathrm{D}_{2}{ }^{+}$ions; a rotational wavepacket is also excited in residual $\mathrm{D}_{2}$ molecules. Both wavepacket types are collapsed a variable time later by an ultrashort probe pulse. We isolate the vibrational wavepacket and quantify its evolution dynamics through theoretical comparison. Requirements for quantum computation (initial coherence and quantum state retrieval) are studied using this well-defined (small number of initial states at room temperature, initial wavepacket spatially localized) singleelectron molecular prototype by temporally stretching the pump and probe pulses.
\end{abstract}

DOI: 10.1103/PhysRevA.76.053402

PACS number(s): 42.50.Hz, 03.67.Lx, 82.53.Hn

As with a broad spectrum of quantum systems, if a molecule laser-induced into a coherent superposition of vibrational states is free to evolve, a time-dependent wavepacket is created. The wavepacket spreads or dephases significantly after the initial superposition, the result of the constraining potential surface anharmonicity. However, when the discrete eigenstates naturally rephase or revive, the original state of the system is almost fully recovered, and the initial quasiclassical periodicity returns. This phenomenon of wavepacket revival [1] has major implications in experimentally realizable time-evolving quantum systems. Through the precise optical preparation of a tailored wavepacket in a molecule, the wavepacket motion is imprinted on the dissociative states, observable with a probability governed by the amplitudes that contribute to the superposition.

In this work, we report the isolation and quantification of a vibrational wavepacket in a room-temperature ensemble of $\mathrm{D}_{2}{ }^{+}$ions. We vary the initial coherence of the superposed states and distinguish a structure in the wavepacket revival when the measurement condition is changed. An application of these results is as a single-internal degree-of-freedom prototype vibrational qubit (vibrational states, $v$, support the qubit), the building block of a molecular quantum computer (MQC). Quantum computation operations are not performed, rather we test the preparation and observation of a superposition. While MQC has been demonstrated in $\mathrm{Li}_{2}[2]$, the $\mathrm{D}_{2}{ }^{+}$ system is considered as it is readily handled theoretically [numerical solution of the rotation-free 1D time-dependent Schrödringer equation (TDSE) under the two-state approximation] [3] and generated experimentally $\left(D_{2}\right.$ molecules at room temperature are predominantly in $v=0$, no stateselective cooling is required; short laser pulse defines a spatially localized initial state) [3-5].

\footnotetext{
*Also at Central Laser Facility, Rutherford Appleton Laboratory, Chilton, Didcot, Oxon OX11 0QX, UK; w.bryan@ucl.ac.uk

†.williams@qub.ac.uk

*w.r.newell@ucl.ac.uk
}

Before implementing quantum computation (QC), five general "physical implementation" requirements have been identified [6]: (1) The ability to initialize the qubit in a fiducial state, (2) the coherence time of the qubit is much longer than the operation times, (3) a universal set of logic operators are available, (4) a qubit-specific measurement capability exists, and (5) the quantum system is well characterized and readily scalable. We quantify the fulfilment of requirements (1), (4), and (5) with a discussion of requirement (2).

Clearly requirement (3) lies at the heart of QC, which in future experiments will be attempted by optically controlling the phase [7] or amplitude [8] of each vibrational state, referred to as phase or amplitude manipulation. The additional degrees of freedom in a larger molecule could be employed [9], i.e., compound vibrational or bending modes, making scalable (multidimensional) QC feasible.

Tunnel ionization $\left[\mathrm{D}_{2}\left(v^{\prime}=0\right)+\right.$ pump $\rightarrow \mathrm{D}_{2}^{+}(v=0,1,2 \ldots)$ $\left.+e^{-}\right]$when the Keldysh parameter $\gamma<1[10]$ creates a vibrational wavepacket on the $1 s \sigma_{g}$ potential surface of $\mathrm{D}_{2}^{+}[3-5]$, and is near-instantaneous so it is treated as a "vertical" transition. The configuration of the potential energy surfaces is such that ionization only transfers population around the inner turning point of the wavepacket motion [3-5]. In a phase manipulation MQC, such a transition fulfills requirement (1), as all $v$ states are initiated with essentially zero phase difference. In an amplitude manipulation MQC, requirement (1) is similarly fulfilled as all accessible $v$-states have a known (Franck-Condon) amplitude distribution [11]. However, in practice an ensemble of molecules may be used to generate a meaningful signal, where it becomes important that the pulse length is sufficiently short to retain coherence in the ensemble.

Observing the vibrational wavepacket evolution on the $1 s \sigma_{g}$ ground potential surface is possible by "collapsing" the wavepacket to an observable state with a second laser pulse. Photodissociation (PD) $\mathrm{D}_{2}{ }^{+}+$probe $\rightarrow \mathrm{D}^{+}+\mathrm{D}$ breaks the internuclear bond via photon coupling the $1 s \sigma_{g}$ ground and $2 p \sigma_{u}$ first excited states [12]. A second route to observing 
wavepacket evolution is through double ionization (DI) $\mathrm{D}_{2}^{+} \rightarrow \mathrm{D}^{+}+\mathrm{D}^{+}+e^{-}$, requiring a higher laser intensity as a second ionization event must occur [12]. PD and DI are distinguishable by the kinetic energy released during bond fracture. Both PD and DI are enhanced when the wavepacket is at the outer turning point $\simeq 3.5$ a.u. [3-5], and the internuclear potential energy surfaces naturally act as a quantum "shutter" or "barrier" depending on whether they are lasercoupled or not.

The experimental setup is an extension of one described elsewhere $[3,4,13]$ : Intense $\left(4 \times 10^{14} \mathrm{~W} \mathrm{~cm}^{-2}\right)$ ultrashort $(12$ femtosecond, $\left.1 \mathrm{fs}=10^{-15} \mathrm{~s}\right)$ near infrared $(800 \mathrm{~nm})$ laser pulses are used to create (pump) and observe (probe) the vibrational wavepacket. A colinear low-dispersion MachZehnder interferometer capable of supporting the pulse bandwidth $(>100 \mathrm{~nm})$ generates the time-separated pump and probe, allowing a delay $0 \leq \Delta t \leq 160$ picoseconds between the pump and probe with a repeatable resolution of $\simeq 300$ attosecond $\left(1 \mathrm{as}=10^{-18} \mathrm{~s}\right)$. An alternative method for generating two or three time-delayed pulses has been demonstrated [14] by transmitting a $\simeq 10$ fs pulse through a diskdouble annulus optic allowing two variable delays of hundreds of fs if the two annuli are radially rotated. After focusing with a spherical mirror, the interaction volumes generated by our Mach-Zehnder configuration overlap perfectly, whereas in the disk-annulus configuration, a timevarying spatial overlap is produced. Furthermore, the dispersion of the Mach-Zehnder configuration does not change with delay. $\mathrm{D}^{+}$fragments from PD and DI, Fig. 1(a), are detected in an ion time-of-flight mass spectrometer containing a $250 \mu \mathrm{m}$ aperture; thus a signal can only result from PD or DI when the molecular axis is parallel to the probe polarization and detector axis. The aperture also defines a quasiisointensity source in the focal volume.

In addition to creating a $\mathrm{D}_{2}{ }^{+}$vibrational wavepacket, fewcycle pulses impulsively align deuterium $[13,14]$, thus the revival of a rotational wavepacket is expected. We isolate the vibrational and rotational motions, which is only possible for a limited range of pump pulse durations as discussed later. As shown in Fig. 1(a), in the region $450 \leq \Delta t \leq 650 \mathrm{fs}$, both PD and DI yields exhibit $\mathrm{a} \simeq 24$ fs revival modulation with maximum contrast at $550 \mathrm{fs}$, the result of vibrational revival, consistent with recent findings [3-5]. However, between $250 \leq \Delta t \leq 350$ fs a rotational revival is also observed, comparable to recent observations $[13,14]$ which will also underlie the vibrational revival around $550 \mathrm{fs}$. Both the rotational and vibrational revivals are evident in the integrated PD yield as a function of $\Delta t$, Fig. 1(b) (top).

A major finding of the present work concerns the separation of these two wavepackets. The isolation of the vibrational wavepacket requires confirmation that two distinct populations in $\mathrm{D}_{2}$ and $\mathrm{D}_{2}{ }^{+}$exist following the pump. In recent work by the authors [13] (see also [14]), we quantify the rotational wavepacket in $\mathrm{D}_{2}$ by Fourier transformation of the high-energy time-dependent DI signal recorded under identical experimental conditions. We observe peaks (with an accuracy of $5 \%)$ in the frequency spectrum at $5.5(0), 9.2(1)$, 12.8 (2), 16.4 (3), 20.1 (4), and $23.7(5) \mathrm{THz}$, where parentheses indicate the $J: J+2$ rotational state beat in the $\mathrm{D}_{2}$ molecule. There is no evidence for a rotational wavepacket in
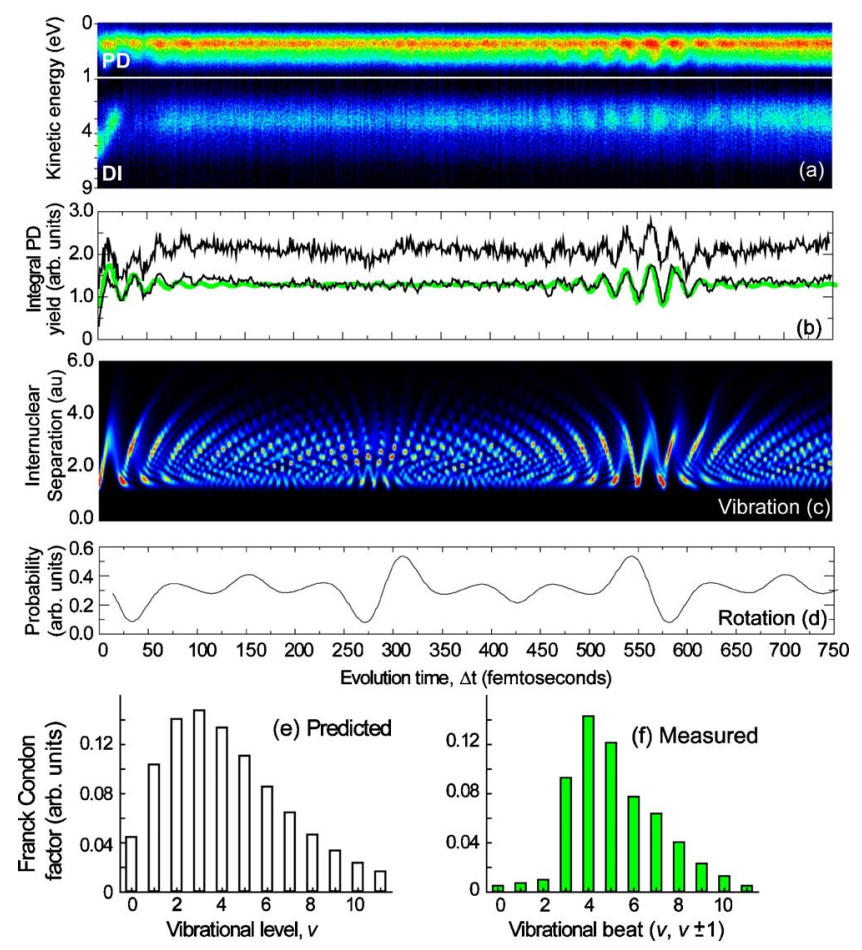

FIG. 1. (Color online) Comparison between experiment and theory showing vibrational and rotational revivals. (a) $\mathrm{D}^{+} \mathrm{PD}$ (top) and DI (bottom) yield as a function of wavepacket evolution time, $\Delta t$, and ion kinetic energy, recorded at room temperature. (b) Integrated PD yield (thick black) contains vibrational and rotational revivals (top). The theoretical prediction [green (thick) gray curve] shows remarkable agreement with the experimental results when rotational dynamics are excluded (thin black curve). (c) The calculated time-dependent probability distribution. (d) The calculated impulsive alignment of $\mathrm{D}_{2}$ induced by the pump pulse in a roomtemperature distribution of rotational levels. (e) The predicted Franck-Condon (FC) distribution. (f) Fourier transform of the experimental PD yield. The frequency components present in the vibrational wavepacket are beats between vibrational states $v$ and $v \pm 1$. The Fourier amplitudes thus return a measure of vibrational population.

$\mathrm{D}_{2}^{+}$, which would contribute peaks in the frequency spectrum at 2.7 (0), 4.5 (1), 6.3 (2), 8.1 (3), 9.9 (4), 11.7 (5), 13.5 (6), 15.3 (7), and 17.1 (8) THz. This is confirmed in the present work. Furthermore, the vibrational wavepacket can only exist in the $\mathrm{D}_{2}{ }^{+}$molecular ion generated in the pump, as the pump duration is greater than the quasiclassical period (11 fs) of $D_{2}$ as recently investigated [15].

Thus we find that the pump either creates a vibrational wavepacket in $\mathrm{D}_{2}{ }^{+}$(rotating in a non-time-correlated manner with respect to the pump with a room-temperature distribution of $J$ states) or a rotational wavepacket in $\mathrm{D}_{2}$ (vibrating incoherently in an impulsively modified distribution of $J$ states). Solving the TDSE for the vibrational [3] [Fig. 1(c)], and rotational [13] [Fig. 1(d)] degrees of freedom, we quantify these motions. We take the integrated PD yield as a function of $\Delta t$ [Fig. 1(b), top], and subtract the probability of finding the rotational wavepacket within the detector acceptance, Fig. 1(d). The integrated PD yield (arb. units) is scaled 
relative to the rotational wavepacket probability until the signature of the rotational wavepacket $(250 \leq \Delta t \leq 350 \mathrm{fs})$ is totally suppressed. The resulting PD yield contains the pure vibrational wavepacket, as shown in Fig. 1(b) (bottom). The PD yield is modeled by numerically propagating the initially localized state (FC principle) then calculating the time dependent coupling of the $1 s \sigma_{g}$ and $2 p \sigma_{u}$ states [3]. The vibrational wavepacket in the molecular ion is clearly welldescribed by this relatively simple treatment; thus requirement (5) is partially fulfilled. If evidence for strong rotational-vibrational coupling had been found, this would not be the case.

Fourier transformation of the raw time-dependent PD yield, Fig. 1(b), recovers the beat frequencies of the vibrational levels with the associated amplitudes that form the vibrational wavepacket, therefore we have a method for recovering the population of the vibrational states. As is apparent from Figs. 1(e) and 1(f), the measured distribution of vibrational beat frequencies agrees with the expected FC distribution of states for $v>4$. For states $v<4$, negligible beating is observed. This is a result of the probe pulse being of insufficient intensity to open the three-photon crossing [12], thus the vibrational wavepacket "below" $v=4$ remains bound. Our "quantum shutter" is therefore selective, however the principle is sound, since given a probe intensity $>6 \times 10^{14} \mathrm{~W} \mathrm{~cm}^{-2}$ all $v$ states will be accessed. It should be noted that the states $0<v<4$ must be populated, otherwise the quasiclassical period of $\mathrm{D}_{2}{ }^{+}$would not be observed: Lack of population in these states would increase the vibrational revival periodicity. In a phase manipulation $\mathrm{MQC}$, the relative phase can be temporally resolved for occupied $v$ states; while in an amplitude manipulation MQC, the relative amplitudes of the constituent $v$ states can also be recovered by Fourier transform analysis, Fig. 1(f). Returning to QC requirement (4), the ability to determine the final state in our measurement is met. Destruction of the prototype qubits is necessary to read the final state, however it is not necessary to destroy the wavepacket to manipulate. By utilizing nondestructive pulses, it should be possible to modify the wavepacket while maintaining the superposition.

To extend our understanding of fulfilling requirements (1) and (4), we quantify variations in the initial vibrational wavepacket coherence. We also study wavepacket distinguishability, the faculty to temporally discern subsequent oscillations of the vibrational wavepacket, particularly around revival. Both are macroscopically achieved through independently varying the pump and probe durations by changing the dispersion (hence chirp) in either arm of the interferometer.

Figure 2(a) shows the integrated PD yield in the vicinity of the vibrational revival as the pump duration, $\tau_{\mathrm{pu}}$, is increased from 14 to $28 \mathrm{fs}$ while the probe duration, $\tau_{\mathrm{pr}}$, is fixed at $13 \mathrm{fs}$. The vibrational wavepacket in $\mathrm{D}_{2}{ }^{+}$was isolated from the rotational signature in $\mathrm{D}_{2}$ as described earlier. The contrast of the vibrational revival structure decreases as the pump duration increases, the consequence of loss of coherence in the initial superposed state. The experimental revival structure [thin black curve, Fig. 2(a)] is compared to the theoretical wavepacket [Fig. 1(c)] averaged over the pump pulse, Fig. 2(b), and integrated $3 \leq R \leq 10$ a.u., then

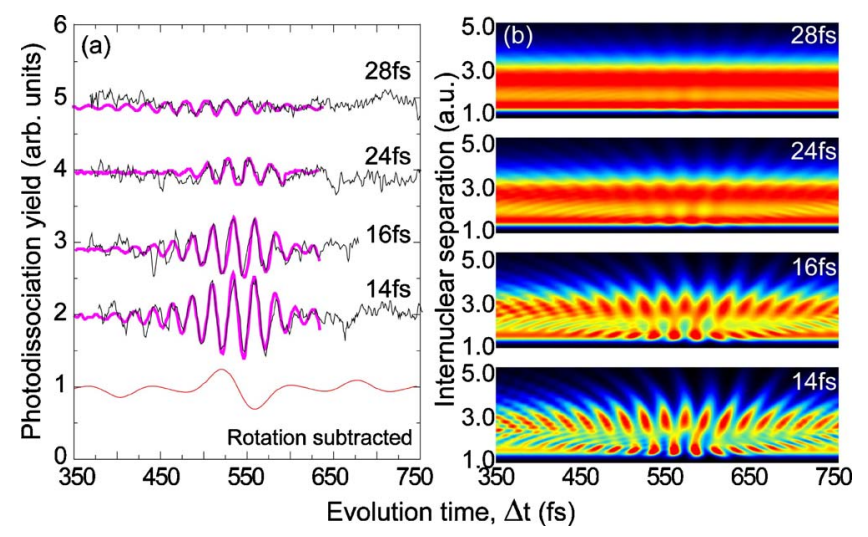

FIG. 2. (Color online) Controlling the initial superposition in an isolated vibrational wavepacket. (a) Experimental PD yield as the pump duration is increased (thin black curve). The rotational wavepacket has been removed from the dataset as discussed. The shape of the revival indicates the superposition degradation as the pump is stretched, illustrated by comparison with a theoretical prediction [thick magenta (gray) curves]. (b) The predicted vibrational wavepacket after averaging over the changing Gaussian pump duration.

overlaid [thick magenta (gray) curves] on the experimental data in Fig. 2(a). The resultant magenta curves show an excellent agreement with the decohering isolated vibrational wavepacket, clearly demonstrating the role of coherence in the initial pump pulse.

In Fig. 2(a), it is apparent that even for $\tau_{\text {pu }}=28 \mathrm{fs}$, some vibrational coherence remains when $\tau_{\mathrm{pu}}$ is longer than the revival oscillatory period, $24 \mathrm{fs}$. This residual structure is due to the highest vibrational states, $v>9$ which have the longest periods and an absolute population of less than $4 \%$. Consequently, care must be taken in defining the initial state of a vibrational qubit as states with an apparently negligible population clearly influence wavepacket evolution.

By increasing $\tau_{\mathrm{pr}}$ from 14 to $60 \mathrm{fs}$ while keeping the pump fixed at $\tau_{\mathrm{pu}}=14$ fs [thus the vibrational wavepacket is identical to Fig. 2(b), bottom, throughout], the amplitude of the revival changes suddenly as $\tau_{\mathrm{pr}}$ exceeds $40 \mathrm{fs}$. Since a 15 fs probe, Fig. 3(b), is short compared to the revival, oscillations are observed with high fidelity. At 30 fs, Fig. 3(c), a comparable fidelity is also achieved, as subsequent revivals are still distinguished. However, a 45 fs probe, Fig. 3(d), cannot distinguish any two revival maxima, hence the oscillatory structure is suddenly lost when $\tau_{\mathrm{pr}}$ approaches double the quasiclassical period of $\mathrm{D}_{2}{ }^{+}$. We can therefore define a wavepacket distinguishability condition, which is less strict than for creation, depending on the measurement regime. If a time-dependent measurement is performed at revival (as required in the phase manipulation), $\tau_{\mathrm{pr}}$ must be shorter than double the quasiclassical period to ensure total wavepacket collapse. Alternatively, to guarantee that all the wavepacket is measured in an energy-resolved scheme (as required in the amplitude manipulation), $\tau_{\mathrm{pr}}$ of at least double the period of the highest populated state is required. Furthermore, the intensity requirements discussed earlier must also be adhered to.

The pump-variation observations indicate a promising future for controlling the initial superposition conditions. Fol- 


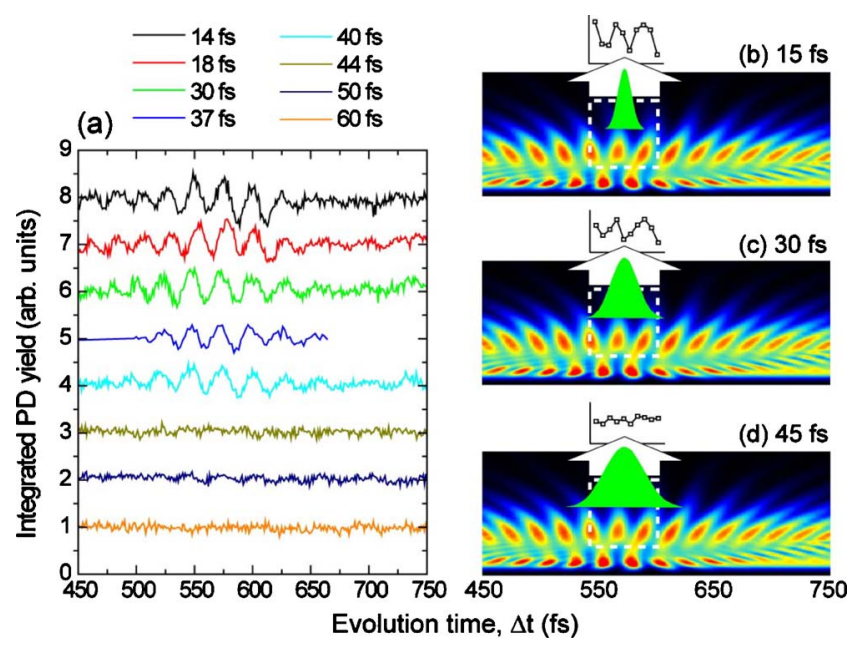

FIG. 3. (Color online) Distinguishing the reviving vibrational wavepacket as the measurement condition is changed. (a) The sudden loss of revival fidelity when the probe duration exceeds $40 \mathrm{fs}$, the consequence of the temporal structure becoming indistinguishable to the probe. From top to bottom, the probe durations are indicated sequentially. Comparing a (b) $15 \mathrm{fs}$, (c) $30 \mathrm{fs}$, and (d) $45 \mathrm{fs}$ probe to the revival structure, the facility to discern subsequent oscillations (i.e., wavepacket distinguishability) of the $\mathrm{D}_{2}{ }^{+}$ vibrational revival is compromised.

lowing the optical preparation of an isolated vibrational wavepacket in a single molecule, a genetic algorithm will determine the intermediate time- and frequency-structured laser pulse [16] required to achieve a particular target state (either by phase or amplitude manipulation). This intermediate "operator" pulse must be low intensity so as not to fracture the internuclear bonds, rather to guide wavepacket evolution. The high intensity ultrashort probe pulse will then fracture the internuclear bonds, allowing the result of the operation to be read.

To perform logic operations and apply successful error correction schemes requires a coherence time $\simeq 10^{4}$ times the interaction time, $t_{I}$, requirement (2). Here, ionization defines $t_{I} \simeq 1.3 \mathrm{fs}$ thus if the wavepacket survives to $\simeq 10 \mathrm{ps}$ with high fidelity, QC is possible. Our measurements have exceeded $2 \mathrm{ps}$, therefore this constraint does not appear technologically limiting. The MQC will be scaled by increasing the number of vibrational modes supporting the qubit, accomplished in a larger molecule. Communication between states will then be controlled optically by distorting the electronic potential surface through the Stark shift.

In a broader application, a pump of a shorter duration than the vibrational motion establishes a time-dependent variation of the refractive index in a Raman-active molecular medium, as demonstrated in $\mathrm{SF}_{6}$ [17]. By employing the vibrational mode of a hydrogenic molecular ion, it may be possible to spectrally broaden and self-compress, potentially to the single-cycle level.

The experiment was carried out at the ASTRA Laser Facility, STFC RAL, UK. The assistance of J. M. Smith, E. J. Divall, K. Ertel, O. Chekhlov, C. J. Hooker, and S. Hawkes is gratefully acknowledged. This work was funded by the Engineering and Physical Sciences Research Council (UK). R.T. acknowledges the Spanish Department of State of Education and Universities, and the European Social Fund.
[1] R. W. Robinet, Phys. Rep. 392, 1 (2004).

[2] J. Vala, Z. Amitay, B. Zhang, S. R. Leone, and R. Kosloff, Phys. Rev. A 66, 062316 (2002).

[3] D. S. Murphy et al., J. Phys. B 40, S358 (2007).

[4] J. McKenna et al., J. Phys.: Conf. Ser. 58, 375 (2007).

[5] Th. Ergler, A. Rudenko, B. Feuerstein, K. Zrost, C. D. Schroter, R. Moshammer, and J. Ullrich, Phys. Rev. Lett. 97, 193001 (2006).

[6] D. P. DiVincenzo, Fortschr. Phys. 48, 771 (2000).

[7] K. F. Lee, D. M. Villeneuve, P. B. Corkum, and E. A. Shapiro, Phys. Rev. Lett. 93, 233601 (2004).

[8] H. Niikura, D. M. Villeneuve, and P. B. Corkum, Phys. Rev. Lett. 92, 133002 (2004).

[9] C. M. Tesch and R. de Vivie-Riedle, Phys. Rev. Lett. 89, 157901 (2002).

[10] V. S. Popov, Phys. Usp. 47, 855 (2004).
[11] X. Urbain, B. Fabre, V. M. Andrianarijaona, J. Jureta, J. H. Posthumus, A. Saenz, E. Baldit, and C. Cornaggia, Phys. Rev. Lett. 92, 163004 (2004).

[12] J. H. Posthumus, Rep. Prog. Phys. 67, 623 (2004).

[13] W. A. Bryan, E. M. L. English, J. McKenna, J. Wood, C. R. Calvert, I. C. E. Turcu, R. Torres, J. L. Collier, I. D. Williams, and W. R. Newell, Phys. Rev. A 76, 023414 (2007).

[14] K. F. Lee et al., J. Phys. B 39, 4081 (2006).

[15] Th. Ergler, B. Feuerstein, A. Rudenko, K. Zrost, C. D. Schroter, R. Moshammer, and J. Ullrich, Phys. Rev. Lett. 97, 103004 (2006).

[16] R. J. Levis, G. M. Menkir, and H. Rabitz, Science 292, 709 (2001).

[17] N. Zhavoronkov and G. Korn, Phys. Rev. Lett. 88, 203901 (2002). 